\title{
PO2-7-7
}

Poster session

\section{Selective placental growth factor (PIGF) inhibition: a novel pharmacological approach in diabetic retinopathy}

\author{
Claudio Bucolo, Francesca Lazzara, Anna Fidilio, Salvatore Salomone, Filippo Drago \\ Biomedical and Biotechnological Sciences, University of Catania, Catania, Italy
}

Background: Diabetic Retinopathy (DR) is one of the most common complications of diabetes and the main cause of vision impairment in the working-age population. Current treatments of DR include intraocular injection of anti-VEGF or steroids despite safety concerns. The aim of the present study was to explore the role of placental growth factor (PlGF) as new pharmacological target in DR.

Methods: Primary human retinal endothelial cells (HREC; Innoprot) were used in this study. We assessed TNF- $\alpha$, VEGF and ERK in HREC exposed (48 h) with high glucose $(40 \mathrm{mM})$ with or without anti-PlGF antibody treatment at different concentrations (from 1 to $50 \mu \mathrm{g} / \mathrm{ml}$ ) by ELISA and Western blot. The effects of anti-PIGF antibody treatment were compared with aflibercept, a VEGF-trap, in order to discriminate the contribution of PIGF inhibition. The viability of HREC was determined using a colorimetric MTT assay. Cellular damage was also assessed by LDH release into the medium.

Results: Anti-PIGF protects HREC against the damage elicited by high glucose. High glucose significantly $(\mathrm{p}<0.01)$ increased the levels of activated/phosphorylated ERK, while anti-P1GF treatment significantly $(\mathrm{p}<0.01)$ counteracted ERK phosphorylation, induced by high glucose, in a concentration-dependent manner. HREC exposed to high glucose increased the TNF- $\alpha(40 \mathrm{pg} / \mathrm{ml})$ and the VEGF $(80 \mathrm{pg} / \mathrm{ml})$ levels that were significantly $(\mathrm{p}<0.01)$ attenuated, in a concentration-dependent manner, by anti-PlGF treatment. No significant effects were observed with aflibercept treatment, except for VEGF levels.

Conclusions: In conclusion, our data indicate that selective inhibition of PlGF protects HREC against high glucoseinduced damage through the inhibition of ERK pathway. Moreover the expression of cytokines such as TNF- $\alpha$ and VEGF were also decreased by anti-PIGF treatment. Such a mechanism, partially distinct from VEGF blockade, if occurring in vivo may contribute to the potential therapeutic effect of anti-PlGF antibody in diabetic retinopathy. 